\title{
Induction of meiotic maturation of follicle-enclosed oocytes of rabbits by a transient increase followed by an abrupt decrease in cyclic AMP concentration
}

\author{
Y. Yoshimura ${ }^{1}$, Y. Nakamura ${ }^{1}$, T. Oda ${ }^{2}$, M. Ando ${ }^{1}$, Y. Ubukata ${ }^{1}$, \\ M. Karube ${ }^{1}$, N. Koyama ${ }^{1}$ and H. Yamada ${ }^{1}$ \\ ${ }^{1}$ Department of Obstetrics and Gynecology, Kyorin University School of Medicine, Tokyo, \\ Japan, 181; and ${ }^{2}$ Department of Obstetrics and Gynecology, Tokyo Dental College, Ichikawa \\ General Hospital, Chiba, Japan, 272
}

\begin{abstract}
Summary. The involvement of cyclic adenosine monophosphate (cAMP) in mammalian oocyte maturation was assessed using cultures of rabbit cumulus-oocyte complexes and perfused rabbit ovaries. Rabbit cumulus-oocyte complexes were cultured in Brackett's medium with or without forskolin at $10^{-4}, 10^{-5}$ or $10^{-6}$ mol 1-1 for 3-6 h. At 3 or 4 h spontaneous meiotic maturation was significantly $(P<0.05)$ inhibited by forskolin at $10^{-4} \mathrm{~mol} \mathrm{l}^{-1}$. With prolonged incubation, spontaneous maturation progressed despite exposure to forskolin. In the second experiment ovaries were perfused for $12 \mathrm{~h}$ with forskolin $\left(10^{-4}, 10^{-5}\right.$ or $\left.10^{-6} \mathrm{~mol} \mathrm{1^{-1 }}\right)$ or medium alone. Neither ovulation nor degeneration of follicular oocytes occurred in any perfused ovary. The percentage of follicular oocytes achieving germinal vesicle breakdown was significantly $(P<0.001)$ increased in response to forskolin in a dose-related manner. In an additional experiment, ovaries were perfused with forskolin at $10^{-4} \mathrm{~mol} 1^{-1}$. A significant increase in the cAMP content in the follicle was observed within $30 \mathrm{~min}$, but the ability to produce cAMP in response to forskolin decreased as the duration of perfusion was increased. Intraoocyte cAMP increased significantly within $30 \mathrm{~min}$ and reached its maximum $2 \mathrm{~h}$ after exposure to forskolin. Thereafter, cAMP levels in the oocytes decreased abruptly. This drop in intraoocyte cAMP concentration was followed by the resumption of meiosis. The alterations of intraoocyte cAMP contents following exposure to $\mathrm{hCG}$ in vivo paralleled those observed in the ovaries perfused with forskolin. These data suggest that a transient, but not continuous, increase in cAMP concentration after the gonadotrophin surge may be required to initiate oocyte maturation.
\end{abstract}

Keywords: oocyte maturation; cAMP; forskolin; gonadotrophin; rabbit

\section{Introduction}

The apparent antagonism between the stimulation by luteinizing hormone (LH) and the inhibition by modulators of cAMP levels in the female gamete is a paradox because a predominant effect of LH is to increase cAMP concentration in preovulatory follicles (Tsafriri et al., 1972; Marsh, 1976). Membrane-permeable cAMP derivatives or phosphodiesterase inhibitors have been shown to inhibit spontaneous oocyte maturation in mammals (Cho et al., 1974; Magnusson \& Hillensjö, 1977; Dekel \& Beers, 1978). Oocyte cAMP originates in the cumulus cells and is transferred to the oocyte via the heterologous gap junctions that exist between the two types of cell. Gonadotrophin stimulation that ultimately terminates cumulus-oocyte communication has been proposed to initiate resumption of meiosis by interrupting the direct transfer of cAMP to the oocyte (Dekel \& 
Beers, 1980). However, a reduction in the intercellular coupling by LH stimulation could not be the trigger to resume meiosis because initiation of meiotic maturation preceded $\mathrm{LH}$-induced termination of the communication (Moor et al., 1981; Eppig, 1982). Increasing the cAMP of the cumulus cell did not result in a detectable rise in oocyte cAMP (Schultz et al., 1983a). A model for the inhibition of meiosis in mice proposed recently that a putative inhibitor of cumulus cell origin is activated by increased cAMP concentration and subsequently passed to the oocyte through the gap junction where it acts to suppress germinal vesicle breakdown (Eppig et al., 1983; Freter \& Schultz, 1984).

Forskolin, a cardioactive diterpene isolated from the roots of Coelus forskohli, activates adenylate cyclase in broken cell preparations as well as in intact tissues and increases intracellular cAMP concentrations (Seamon \& Daly, 1981; Seamon et al., 1981). This diterpene has recently been reported to inhibit the spontaneous maturation of denuded or cumulus-enclosed oocytes in mammals (Schultz et al., 1983b; Dekel et al., 1984; Racowsky, 1984). However, forskolin has been shown to induce meiotic maturation of follicle-enclosed oocytes in rats (Dekel \& Sherizly, 1983). Thus, further studies are needed to clarify the role of cAMP in the physiological regulation of mammalian oocytes. The in vitro perfused rabbit ovary is an extracorporeal model that allows the intrafollicular environment of the oocyte to be controlled for study (Yoshimura et al., 1985, 1986; Holmes et al., 1986). In this study the ovary preparation perfused in vitro was used to examine the effect of forskolin on the meiotic resumption of rabbit oocytes in relation to cAMP accumulation.

\title{
Materials and Methods
}

\begin{abstract}
Animals
Sexually mature Japanese white rabbits were used in all experiments. Animals were isolated for a minimum of 3 weeks before the experimental procedure and were caged individually under controlled light and temperature with free access to Purina Rabbit Chow and water. Rabbits weighing at least $3.5 \mathrm{~kg}$ were anaesthetized with intravenous sodium pentobarbital ( $32 \mathrm{mg} \mathrm{kg} \mathrm{kg}^{-1}$ body weight), anticoagulated with heparin sulfate $\left(120 \mathrm{units}^{\mathrm{kg}}{ }^{-1}\right)$, and then subjected to laparotomy. Mature ovaries of normal appearance were used in the experiments.
\end{abstract}

\section{Culture of isolated oocytes}

A total of 36 ovaries was used in the experiment of in vitro maturation of cumulus-enclosed oocytes. Ovaries were removed and placed in Brackett's medium (Brackett \& Oliphant, 1975) under a dissecting microscope. Follicles greater than $1.0 \mathrm{~mm}$ in diameter were punctured using a 26 -gauge needle and the contents of the follicle were aspirated. Cumulus-oocyte complexes of uniform size were selected and rinsed three times in fresh medium. Cumulusenclosed oocytes were then cultured in Brackett's medium with or without different concentrations of forskolin (Calbiochem Behring, La Jolla, CA) at $37^{\circ} \mathrm{C}$ and $100 \%$ humidity using $5 \% \mathrm{CO}_{2}$ in air for $3-6 \mathrm{~h}$. A stock solution of forskolin was prepared in $95 \%$ ethanol. The concentration of ethanol did not exceed $5 \mu \mathrm{ml}^{-1}$, and an equivalent volume of ethanol was added to the control medium. The maturity of individual oocytes was examined at 3,4 or $6 \mathrm{~h}$ of culture after isolation.

\section{Perfusion system}

Each ovarian artery was cannulated in situ, and the ovary, the ovarian artery, vein and supportive adipose tissue were then removed from the rabbit and immediately placed in a perfusion chamber. Ovaries were perfused with tissue culture medium 199 (M.A. Bioproducts, Walkerville, MD) supplemented with insulin, heparin sulfate, streptomycin and penicillin. The perfusion technique and surgical procedure, including arterial cannulation and ovarian removal, were previously described in detail (Lambertsen et al., 1976; Yoshimura et al., 1987). The perfusate of one ovary contained forskolin dissolved in $95 \%$ ethanol to yield a final concentration of $10^{-4}, 10^{-5}$, or $10^{-6} \mathrm{~mol} 1^{-1}$. The contralateral ovary served as a control and was perfused in the presence of a corresponding concentration of ethanol (less than $0.2 \%$ in the perfusate). Both ovaries were perfused simultaneously for $12 \mathrm{~h}$. Six rabbits were used for each concentraton of forskolin. Ovaries were observed periodically for evidence of follicle growth and rupture. At the end of perfusion, mature follicles that were greater than $1.5 \mathrm{~mm}$ in diameter were punctured and aspirated to recover follicular oocytes for microscopic evaluation. Perfusate samples ( $3 \mathrm{ml})$ were withdrawn at $0,1,2,4,6,8$ and $12 \mathrm{~h}$ after the onset of perfusion. 


\section{Examinations of oocytes}

After culture and perfusion of isolated oocytes the oocytes were fixed and stained with $1 \%$ lacmoid in $45 \%$ acetic acid for microscopy. Oocytes were assessed for stage of maturity and signs of degeneration as described previously (Yoshimura et al., 1990). The degree of oocyte maturity was expressed as the percentage of oocytes that had achieved germinal vesicle breakdown. Oocytes were also assessed for any degenerative change such as vacuolation, cytolysis, necrosis, cytoplasmic flocculation, karyorrhexis or disintegration.

\section{Cyclic AMP alterations in the follicle and oocyte}

In the perfused rabbit ovaries, intrafollicular cAMP accumulated to its maximum $1 \mathrm{~h}$ after exposure to forskolin. Ovaries were perfused with or without forskolin at a concentration of $10^{-3}$ to $10^{-7} \mathrm{~mol} 1^{-1}$ for $1 \mathrm{~h}$ to determine the effect of forskolin on cAMP formation in the follicle. Cyclic AMP was measured in mature follicles greater than $1.5 \mathrm{~mm}$ in diameter after $1 \mathrm{~h}$ of perfusion. The effect of forskolin on the intrafollicular cAMP concentration was examined using a total of 48 ovaries. In the subsequent experiment, the time-dependent accumulation of cAMP in the follicles and oocytes was determined in response to forskolin treatment using a total of 268 ovaries. Increases in the concentrations of cAMP in follicles and oocytes induced by forskolin at $10^{-4} \mathrm{~mol}^{-1}$ were examined at the onset of perfusion and at $0 \cdot 5,1,2,4,6,8$, and $12 \mathrm{~h}$ after the onset of perfusion. The alteration of cAMP content in the follicles derived from forskolin-treated ovaries was also compared with that in ovaries perfused with $50 \mathrm{iu}$ of human chorionic gonadotrophin (hCG). In the final experiment using a total of 92 rabbits, 100 iu of hCG was administered intravenously to determine a series of intraoocyte cAMP contents after the induction of maturation in vivo. The relationship between the intraoocyte cAMP concentrations and the percentage of oocytes achieving germinal vesicle breakdown after exposure to hCG in vivo was compared with that in ovaries perfused with forskolin at $10^{-4} \mathrm{~mol}^{-1}$.

\section{Cyclic AMP determinations}

The cAMP contents of the follicles and oocytes in ovaries perfused with forskolin or in hCG-treated ovaries were determined using the Yamasa cyclic nucleotide assay kit (Yamasa Shoyu Co., Ltd, Chiba, Japan) as previously described (Kunitada et al., 1978; Oda et al., 1990). The follicles were excised and frozen immediately in liquid nitrogen. The sample was stored in liquid nitrogen until cAMP was measured by radioimmunoassay. The follicular tissue was homogenized with $1 \mathrm{ml}$ of cold $6 \%$ trichloroacetic acid (TCA) using a glass homogenizer. After centrifugation, the TCA extract was washed four times with ether that was saturated with water. The supernatants were removed and dried down under a stream of air in a water-bath at $75^{\circ} \mathrm{C}$. The cyclic nucleotide isolated from tissues $(0.1 \mathrm{ml})$ was succinylated by adding $0.1 \mathrm{ml}$ of dioxane which contained succinic anhydride and triethylamine. Samples were then mixed with $0.1 \mathrm{ml}$ of ${ }^{125} \mathrm{I}$-labelled succinyl cAMP tyrosine methyl ester and $0.1 \mathrm{ml}$ of diluted anti-cAMP antiserum. Two different aliquots of each dissolved ovarian extract were measured in duplicate. The sensitivity of this assay was $10 \mathrm{fmol}$ per tube.

At the end of the perfusions of various duration after exposure to hCG in vivo, the cumulus-enclosed oocytes were collected in a minimum volume of medium 199 containing $0.1 \mathrm{mmol} 3$-isobutyl-1-methylxanthine $1^{-1}$ (IBMX, Sigma Chemical Co., St Louis, MO). Cumulus cells were removed from oocytes with a solution of $0.5 \mathrm{mg}$ hyaluronidase $\mathrm{ml}^{-1}$ and gentle agitation with a narrow-bore pipette. Ten to twenty oocytes were transferred into polypropylene tubes in $20 \mu \mathrm{l}$ of $1 \mathrm{~mol} \mathrm{HCl}{ }^{-1}$. Repeated freezing and thawing (three times) of the oocytes in liquid nitrogen was used to break the membranes and release the cAMP content into the $\mathrm{HCl}$ solution. A similar volume of $\mathrm{HCl}$ solution without the oocytes was treated to obtain control values for intraoocyte cAMP determinations. Each sample was succinylated by adding $20 \mu \mathrm{l}$ of dioxane that contained succinic anhydride and triethylamine. After $10 \mathrm{~min}$ at room temperature the mixture was diluted with $60 \mu \mathrm{l}$ of imidazole buffer. These solutions served as the samples of intraoocyte cAMP determination for radioimmunoassay. The sensitivity of this assay was 1 fmol per tube.

\section{Statistical analysis}

Data for the maturity of cumulus-enclosed oocytes and the concentrations of progesterone and cAMP are presented as the mean \pm SEM. Statistical significance was determined using analysis of variance (ANOVA). The $\chi$ squared test with Yates' correction was used to assess per cent degeneration and per cent germinal vesicle breakdown of follicular oocytes.

\section{Results}

\section{Oocyte maturation in cultured cumulus-enclosed oocytes}

The nuclear structure of cumulus-enclosed oocytes of rabbits began to disappear after $3-4 \mathrm{~h}$ of incubation, indicating resumption of meiosis. By $6 \mathrm{~h}$ of incubation, most of the oocytes cultured in 
control medium (93.8\%) showed evidence of meiotic resumption with germinal vesicle breakdown. In contrast, the exposure of cumulus-enclosed oocytes to forskolin prevented the resumption of meiosis (Fig. 1). Furthermore, forskolin inhibited spontaneous oocyte maturation in a dosedependent manner. At $4 \mathrm{~h}$ of incubation, meiotic arrest was observed in $68.8 \%$ and $48.1 \%$ of cumulus-enclosed oocytes in the presence of forskolin at $10^{-4} \mathrm{~mol}^{-1}$ and $10^{-5} \mathrm{~mol} \mathrm{1}^{-1}$, respectively. However, this inhibition was attenuated as the duration of incubation increased. By $6 \mathrm{~h}$ of incubation, $83.8 \%$ of the oocytes had resumed meiosis despite the presence of $10^{-4} \mathrm{~mol}$ forskolin $1^{-1}$. No difference was observed in the percentage of follicular oocytes displaying germinal vesicle breakdown when forskolin-treated and control groups were compared after incubation for $6 \mathrm{~h}$ at each dose.

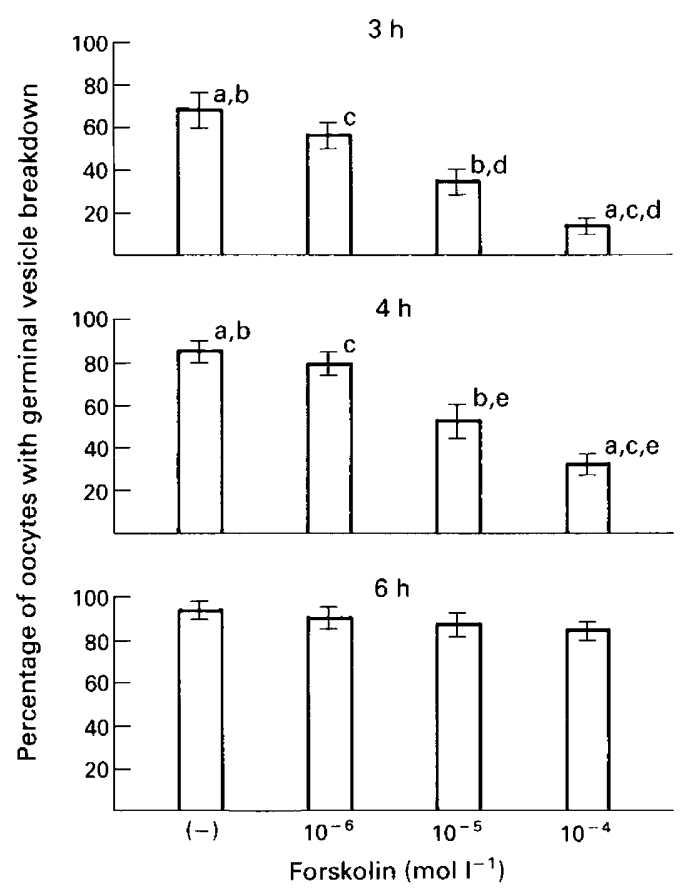

Fig. 1. Effect of forskolin on the spontaneous maturation of cumulus-enclosed oocytes. Isolated cumulus-enclosed oocytes were cultured with or without forskolin. Oocytes were examined after 3,4 and $6 \mathrm{~h}$ of incubation. Data are expressed as the mean \pm SEM of at least six different experiments. Values with the same letters are significantly different: ${ }^{\mathrm{a}, \mathrm{b}} P<0.005 ;{ }^{\mathrm{c}} P<0.01 ;{ }^{\mathrm{d}} P<0.02 ;{ }^{\mathrm{e}} P<0.05$.

\section{Oocyte maturation in the perfused rabbit ovary}

Ovulation did not occur in rabbit ovaries during $12 \mathrm{~h}$ of perfusion with forskolin at concentrations of $10^{-4}, 10^{-5}$ or $10^{-6} \mathrm{~mol} 1^{-1}$ in the absence of gonadotrophin stimulation. The percentage of follicular oocytes achieving germinal vesicle breakdown was significantly $(P<0.001)$ increased in response to forskolin at a concentration of $10^{-4} \mathrm{~mol} \mathrm{l}^{-1}$ compared with controls $(83.8 \%$ germinal vesicle breakdown with forskolin; $5.6 \%$ germinal vesicle breakdown in controls). Furthermore, forskolin at a concentration of $10^{-5}$ or $10^{-6} \mathrm{~mol}^{-1}$ also significantly increased the meiotic maturation of follicular oocytes. Forskolin enhanced the meiotic maturation of follicle-enclosed oocytes in a dose-dependent manner (Fig. 2). Although the percentage of follicular oocytes that showed evidence of degeneration was increased in forskolin-treated ovaries 
at all concentrations, compared with each group of control ovaries, these differences were not significant (forskolin $10^{-4} \mathrm{~mol} \mathrm{1} 1^{-1} 18.9 \%, 10^{-5} \mathrm{~mol} \mathrm{l}^{-1} 25 \cdot 0 \%, 10^{-6} \mathrm{~mol} \mathrm{l}^{-1} 17 \cdot 6 \%$, control $15 \cdot 2 \%$ ).

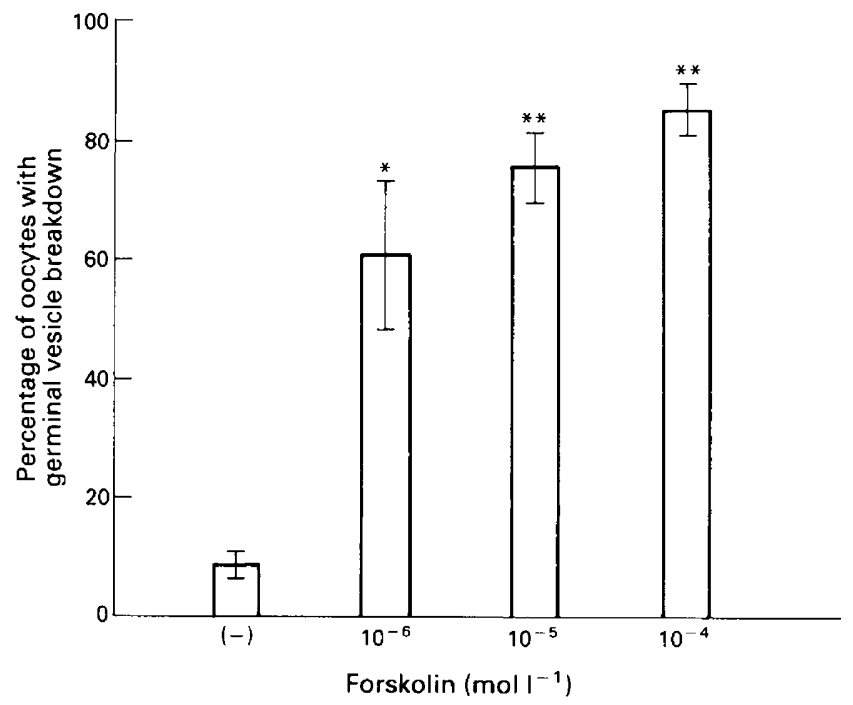

Fig. 2. Percentage of follicular oocytes achieving germinal vesicle breakdown after $12 \mathrm{~h}$ of perfusion with medium alone or with forskolin at a concentration of $10^{-4} \mathrm{~mol}^{-1}$, $10^{-5} \mathrm{moll}^{-1}$ or $10^{-6} \mathrm{moll}^{-1}$. Data are the mean of six different ovaries from at least four experiments. Values with asterisks differ significantly from control ovaries perfused with medium alone: ${ }^{*} P<0.005 ;{ }^{*} P<0.001$.

\section{Cyclic AMP content of follicles}

A significant increase in the cAMP content of the follicle was observed in the forskolinstimulated perfused ovary. Treatment with forskolin enhanced cAMP accumulation in the follicle in a dose-dependent manner (Fig. 3). The maximal effective dose of forskolin was attained at a concentration of $10^{-4} \mathrm{~mol} \mathrm{l}^{-1}$. Forskolin at a concentration of $10^{-4} \mathrm{~mol} \mathrm{l}^{-1}$ in the perfusate increased the cAMP concentration in follicular tissue within $30 \mathrm{~min}$ (Fig. 4). Cyclic AMP accumulation reached a maximum $1 \mathrm{~h}$ after exposure to forskolin. A decrease in the ability of the rabbit follicles to respond to forskolin in vitro was observed as early as $2 \mathrm{~h}$ after the onset of perfusion. At $4 \mathrm{~h}$ after exposure to forskolin, the perfused rabbit follicles could generate only half of the maximum concentration of cAMP. The concentration of follicular cAMP returned to the basal concentration observed in unstimulated ovaries by $12 \mathrm{~h}$ of perfusion. Thus, the production of cAMP in response to forskolin was attenuated as the duration of perfusion increased. Similarly, addition of hCG to the perfusate significantly increased the intrafollicular cAMP level. However, the concentration of intrafollicular cAMP differed significantly between forskolin-treated and hCG-treated ovaries at $0 \cdot 5,1,2$ and $4 \mathrm{~h}$ of perfusion.

\section{Relationship between cAMP content and meiotic maturation of oocytes}

Meiotic maturation of the follicle-enclosed oocytes was initiated $4 \mathrm{~h}$ after exposure to forskolin at $10^{-4} \mathrm{~mol} \mathrm{l}^{-1}$ (Fig. 5). The stimulation of germinal vesicle breakdown in follicle-enclosed oocytes reached its plateau after $8 \mathrm{~h}$ of perfusion. The intracellular content of cAMP in oocytes derived from unstimulated ovarian follicles was $0.36 \pm 0.08 \mathrm{fmol}$ per oocyte. The intraoocyte cAMP content increased significantly within $30 \mathrm{~min}$ and reached its maximum $(4.9 \pm 1.3 \mathrm{fmol}$ per oocyte $) 2 \mathrm{~h}$ 

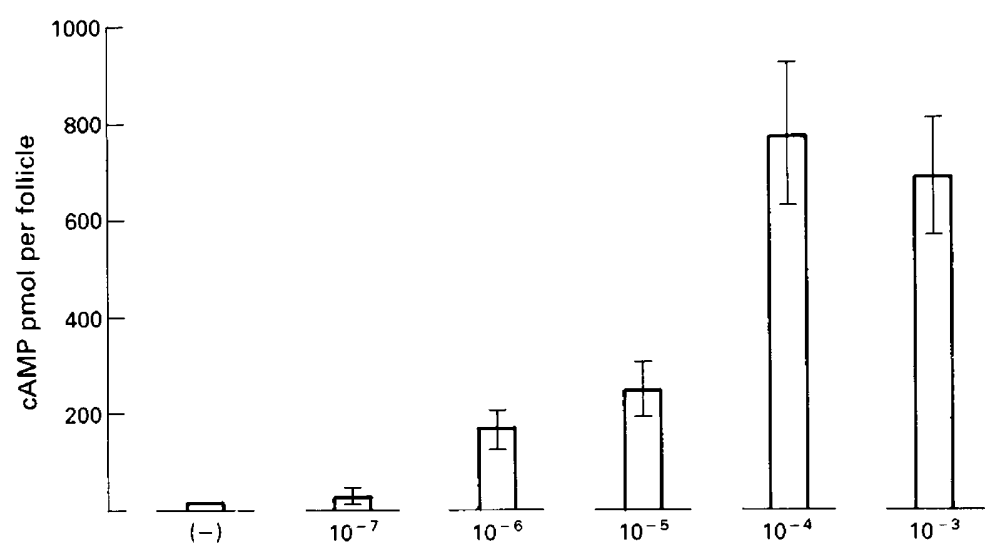

Forskolin $\left\{\mathrm{mol} \mathrm{I}^{-1}\right\}$

Fig. 3. Effect of forskolin on intrafollicular cAMP content. Ovaries were perfused with or without forskolin. Cyclic AMP levels in mature follicles greater than $1.5 \mathrm{~mm}$ in diameter were determined after $1 \mathrm{~h}$ of perfusion. Values are expressed as the mean \pm SEM. Six ovaries were studied for each dose of forskolin.

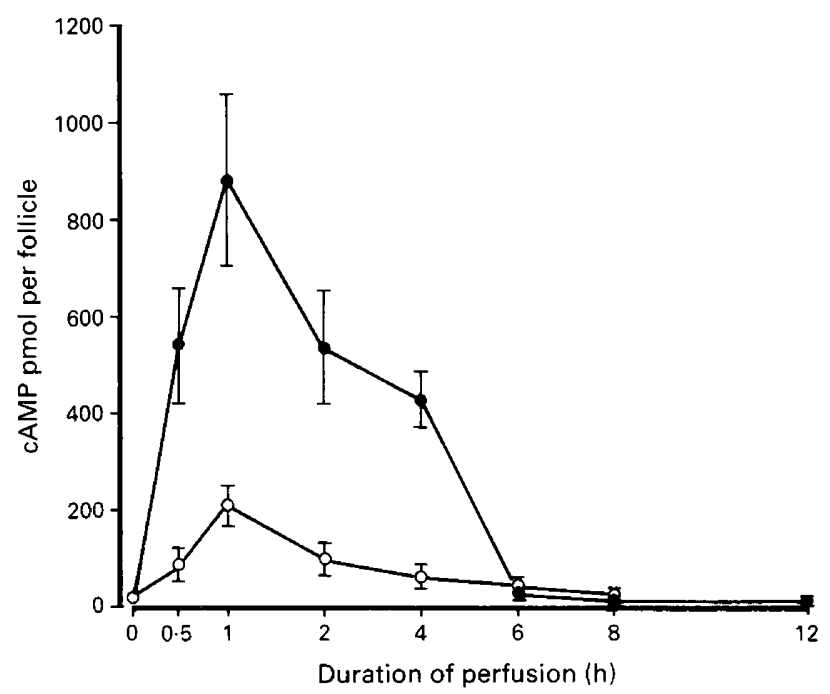

Fig. 4. Changes in cAMP content of follicles during $12 \mathrm{~h}$ of perfusion. Ovaries were perfused with forskolin at a concentration of $10^{-4} \mathrm{moll}^{-1}(-)$ ) or with $50 \mathrm{iu}$ of human chorionic gonadotrophin $(\mathrm{hCG})(\bigcirc-O)$. At least four ovaries were examined at each interval for each treatment. Values are expressed as the mean \pm SEM.

after exposure to $10^{-4}$ mol forskolin $1^{-1}$. Thereafter, the cAMP content of oocytes decreased abruptly and returned to the pretreatment value $6 \mathrm{~h}$ after forskolin administration. This fall in the intraoocyte cAMP content of follicle-enclosed oocytes was followed by their meiotic maturation. After exposure to hCG in vivo, the meiotic maturation of oocytes in the follicles was detected initially within $2 \mathrm{~h}$ (Fig. 6). Although the time course of oocyte maturation in the follicles exposed to hCG in vivo was earlier than that in ovaries perfused with forskolin, the percentage of oocytes achieving germinal vesicle breakdown did not differ significantly between the two groups at each 
time point after exposure to the stimulus. Furthermore, the alterations of intraoocyte cAMP concentrations after exposure to hCG in vivo paralleled those observed in the ovaries perfused with forskolin.

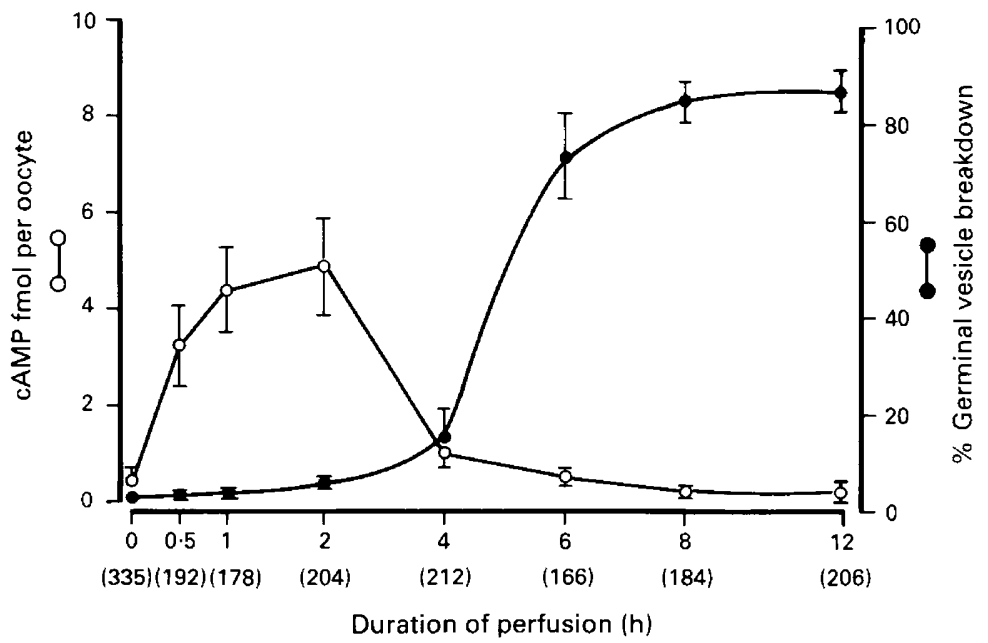

Fig. 5. Relationship between the alteration of intraoocyte cAMP content and meiotic maturation of follicle-enclosed oocytes in ovaries perfused with forskolin. Ovaries were perfused with $10^{-4} \mathrm{~mol}^{-1}$ forskolin. The maturity of oocytes and intraoocyte cAMP content were examined at various times after perfusion of ovaries. The results are presented as mean \pm SEM of at least six replicates of three different experiments. Values in parentheses represent the numbers of oocytes analysed.

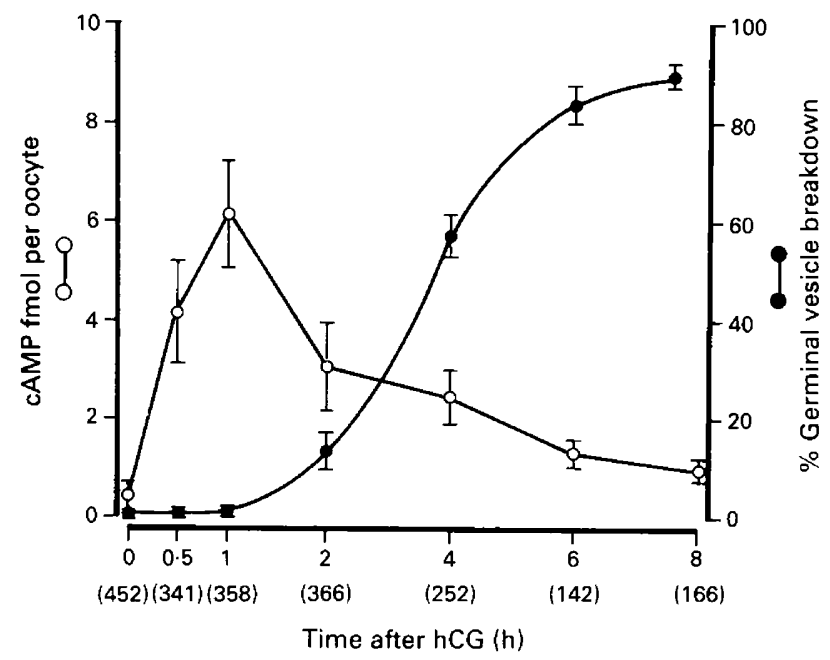

Fig. 6. Relationship between changes in intraoocyte cAMP content and meiotic maturation of follicle-enclosed oocytes in ovaries exposed to human chorionic gonadotrophin (hCG) in vivo. The maturity of oocytes and intraoocyte cAMP content were examined at various times after administration of hCG (100 iu) in vivo. The results are presented as means \pm sEM of at least six replicates of three different experiments. Values in parentheses represent the numbers of oocytes analysed. 


\section{Discussion}

Data derived from culture of cumulus-enclosed oocytes with forskolin are consistent with a previous report that dibutyryl cAMP inhibits the spontaneous maturation of rabbit oocytes (Hosoi et al., 1989). However, most cumulus-enclosed oocytes cultured with forskolin resumed meiosis as the incubation time was increased. Treatment of oocytes with forskolin resulted in a transient and reversible block of the resumption of meiosis in vitro. Since a decrease in cAMP in oocytes is known to correlate with the resumption of meiosis (Schultz et al., 1983b), an alternative explanation would be that the cAMP content of cumulus-enclosed oocytes may decrease with increasing time of incubation. These observations suggest that cAMP may play a significant role in the intrafollicular arrest of oocyte meiosis in rabbits as it does in other mammalian oocytes (Cho et al., 1974; Dekel \& Beers, 1978; Freter \& Schultz, 1984; Racowsky, 1985). However, the inhibition by cAMP of oocyte maturation has been demonstrated only with data from experiments studying the spontaneous maturation of cumulus-enclosed and denuded oocytes (Cho et al., 1974; Magnusson \& Hillensjö, 1977; Dekel \& Beers, 1978, 1980; Freter \& Schultz, 1984; Aberdam et al., 1987).

Dekel et al. (1988) recently showed that cAMP has a dual role in regulating oocyte maturation. Lower concentrations of the nucleotide act to maintain meiotic arrest whereas higher concentrations mediate gonadotrophin-induced resumption of meiosis. In the follicle, however, the possibility that a transient rise in CAMP is necessary for initiation of meitoic maturation of follicleenclosed oocytes has previously been reported (Ekholm et al., 1984; Hubbard, 1985). Forskolin, which activates adenylate cyclase, has been shown to mimic the effects of gonadotrophin on the follicle, stimulating both cAMP accumulation and oocyte maturation in rats and hamsters (Dekel \& Sherizly, 1983; Hubbard, 1985). Although spontaneously maturing oocytes serve as useful models for studying oocyte maturation, there is evidence suggesting that follicle-enclosed oocytes behave in a physiologically different manner from cumulus-enclosed and denuded oocytes when stimulated by LH in vitro (Moor et al., 1981; Ekholm et al., 1984; Hubbard, 1985). Conclusions regarding the role of cAMP drawn from data in experiments that study the spontaneous maturation of cumulus-enclosed and denuded oocytes may require critical evaluation for the physiological regulation of maturation of mammalian oocytes.

The perfused rabbit ovary has been used to evaluate the effects of specific phenomena associated with oocyte maturation while the microenvironment of oocytes is controlled (Yoshimura et al., 1985, 1986; Hosoi et al., 1989). Exposure of the perfused rabbit ovary to forskolin accelerated the resumption of meiosis in follicle-enclosed oocytes in proportion to the accumulation of cAMP in the preovulatory follicles. These results are supported by the observation that forskolin can induce maturation of follicle-enclosed oocytes in rats (Dekel \& Sherizly, 1983; Ekholm et al., 1984). We demonstrated earlier that exposure to dibutyryl cAMP for $2 \mathrm{~h}$ in the two-step perfusion model significantly accelerated meiotic maturation in a dose-related manner. However, this stimulatory effect was not observed when follicle-enclosed oocytes were continuously exposed to dibutyryl cAMP during $12 \mathrm{~h}$ of perfusion or cultured with medium alone in the absence of dibutyryl cAMP (Hosoi et al., 1989). The initiation of meiotic maturation could be observed only after removing dibutyryl cAMP from the perfusate, thus avoiding the inhibition by continuous exposure to dibutyryl cAMP. These data imply that high, sustained concentrations of cAMP are responsible for the maintenance of meiotic arrest but exposure to high level of cAMP is a prerequisite for initiation of meiotic maturation.

Forskolin increased cAMP formation of preovulatory rabbit follicles. The increase in both intrafollicular and intraoocyte cAMP concentrations within $30 \mathrm{~min}$ of exposure to forskolin was followed by a rapid decline at $4 \mathrm{~h}$. The fall in the intraoocyte cAMP concentration resulted in the initiation of meiotic maturation of follicle-enclosed oocytes. The similar relationships between the intraoocyte CAMP concentrations and the percentage of oocytes achieving germinal vesicle breakdown were observed in ovaries perfused with forskolin in vitro and in ovaries exposed to hCG in 
vivo. These data clearly demonstrated that forskolin can mimic the effects of hCG, stimulating both the intracellular cAMP content and the meiotic maturation of follicle-enclosed oocytes. All doses of forskolin that stimulated intrafollicular cAMP synthesis also accelerated the resumption of meiosis. The ability of forskolin to accelerate the resumption of meiosis in a dose-dependent manner suggests that increased cAMP concentrations may be a signal for the resumption of meiosis rather than being responsible for intrafollicular arrest. Gonadotrophin-induced maturation in follicle-enclosed oocytes may be a cAMP-mediated phenomenon.

The results of this study may be applied to resolve the paradoxical observation that cAMP inhibits the spontaneous maturation of cumulus-enclosed oocytes, whereas the gonadotrophin surge stimulates both the formation of cAMP in preovulatory follicles and the resumption of meiosis in follicle-enclosed oocytes. Continuously high cAMP concentrations inhibit oocyte maturation in both cumulus-enclosed and follicle-enclosed oocytes. The ability to accelerate the resumption of meiosis is not observed when oocytes, regardless of whether they are enclosed by cumulus or follicles, are continuously exposed to high cAMP concentration. In contrast, transient exposure of the preovulatory follicles to a high cAMP concentration accelerates the resumption of meiosis in follicle-enclosed oocytes. The initiation of meiotic maturation can be observed when the cAMP concentration in the intrafollicular environment is reduced abruptly after a transient increase. The inhibition of cumulus-enclosed oocytes by forskolin is reversible and most oocytes achieve germinal vesicle breakdown after incubation for $6 \mathrm{~h}$. Alternatively, increases in both intrafollicular and intraoocyte cAMP concentrations may be an essential prerequisite for the resumption of meiosis during the process of ovulation.

In conclusion, we found that forskolin postponed the spontaneous maturation in vitro of cultured cumulus-enclosed oocytes in rabbits in a similar way to that observed in other mammals. In perfused rabbit ovaries, doses of forskolin that stimulate intrafollicular cAMP synthesis also accelerate the resumption of meiosis. Furthermore, forskolin can mimic the effects of gonadotrophin, stimulating both the intracellular cAMP content and meiotic maturation of follicle-enclosed oocytes. These data provide evidence that changes in cAMP concentrations that follow the gonadotrophin surge play a significant role in the resumption of meiosis in follicleenclosed oocytes. A transiently increased intrafollicular cAMP concentration may be an essential prerequisite for meiotic maturation during ovulation.

\section{References}

Aberdam, E., Hanski, E. \& Dekel, N. (1987) Maintenance of meiotic arrest in isolated rat oocytes by the invasive adenylate cyclase of Bordetella pertussis. Biology of Reproduction 36, 530-535.

Brackett, B.G. \& Oliphant, G. (1975) Capacitation of rabbit spermatozoa in vitro. Biology of Reproduction 12, $260-274$.

Cho, W.K., Stern, S. \& Biggers, J.D. (1974) Inhibitory effect of dibutyryl cAMP on mouse oocyte maturation in vitro. Journal of Experimental Zoology 187, 383-386.

Dekel, N. \& Beers, W.H. (1978) Rat oocyte maturation in vitro: relief of cyclic AMP. Proceedings of National Academy of Sciences USA 75, 4369-4373.

Dekel, N. \& Beers, W.H. (1980) Development of the rat oocyte in vitro: inhibition of maturation in the presence or absence of the cumulus oophorus. Developmental Biology 75, 247-254.

Dekel, N. \& Sherizly, I. (1983) Induction of maturation in rat follicle-enclosed oocyte by forskolin. FEBS Letters 151, 153-155.

Dekel, N., Aberdam, E. \& Sherizly, I. (1984) Spontaneous maturation in vitro of cumulus-enclosed rat oocytes is inhibited by forskolin. Biology of Reproduction 31, 244-250.

Dekel, N., Galiani, D. \& Sherizly, I. (1988) Dissociation between the inhibitory and the stimulatory action of cAMP on maturation of rat oocytes. Molecular and Cellular Endocrinology 56, 115-121.

Ekholm, C., Hillensjö, T., Magnusson, C. \& Rosberg, S. (1984) Stimulation and inhibition of rat oocyte meiosis by forskolin. Biology of Reproduction $\mathbf{3 0}$, $537-543$.

Eppig, J.J. (1982) The relationship between cumulus celloocyte coupling, oocyte meiotic maturation, and cumulus expansion. Developmental Biology 89, $268-272$.

Eppig, J.J., Freter, R.R., Ward-Bailly, P.F. \& Schultz, R.M. (1983) Inhibition of oocyte maturation in the mouse: participation of cAMP, steroid hormones, and a putative maturation-inhibitory factor. Developmental Biology 100, 39-49.

Freter, R.R. \& Schultz, R.M. (1984) Regulation of murine oocyte meiosis: evidence for a gonadotropininduced, cAMP-dependent reduction in a maturation inhibitor. Journal of Cell Biology 98, 1119-1128. 
Holmes, P.V., Hedin, L. \& Janson, P.O. (1986) The role of cyclic adenosine $3^{\prime}, 5^{\prime}$-monophosphate in the ovulatory process of the in vitro perfused rabbit ovary. Endocrinology 118, 21952202.

Hosoi, Y., Yoshimura, Y., Atlas, S.J., Adachi, T. \& Wallach, E.E. (1989) Effects of dibutyryl cyclic AMP on oocyte maturation and ovulation in the perfused rabbit ovary. Journal of Reproduction and Fertility 85, 405-411.

Hubbard, C.J. (1985) The effects of forskolin and LH on cAMP changes and maturation in the follicleenclosed oocytes of hamsters. Acta Endocrinologica $110,413-420$.

Kunitada, S., Honma, M. \& Ui, M. (1978) Increases in plasma cyclic AMP dependent on endogenous catecholamines. European Journal of Pharmacology 48, 159-169.

Lambertsen, C.J., Jr, Greenbaum, D.F., Wright, K.H. \& Wallach, E.E. (1976) In vitro studies of ovulation in the perfused rabbit ovary. Fertility and Sterility 27 , $178-187$.

Magnusson, C. \& Hillensjö, T. (1977) Inhibition of maturation and metabolism in rat oocytes by cyclic AMP. Journal of Experimental Zoology 201, $139-147$.

Marsh, J.M. (1976) The role of cyclic AMP in gonadal steroidogenesis. Biology of Reproduction 14, 30-53.

Moor, R.M., Osborn, J.C., Cran, D.G. \& Walter, D.E. (1981) Selective effect of gonadotropins on cell coupling, nuclear maturation and protein synthesis in mammalian oocytes. Journal of Embryology and Experimental Morphology 61, 347-367.

Oda, T., Yoshimura, Y., Izumi, Y., Yoshimura, S., Hara, T., Takehara, Y., Nakamura, Y. \& Ohno, T. (1990) The effect of the follicular fluid adenosine $3^{\prime}, S^{\prime}$-monophosphate degradation rate on successful fertilization and cleavage of human oocytes. Journal of Clinical Endocrinology and Metabolism 71, 116-121.

Racowsky, C. (1984) Effect of forskolin on the spontaneous maturation and cyclic AMP content of rat oocyte-cumulus complexes. Journal of Reproduction and Fertility 72, 107-116.

Racowsky, C. (1985) Effect of forskolin on maintenance of meiotic arrest and stimulation of cumulus expansion, progesterone and cyclic AMP production by pig oocyte-cumulus complexes. Journal of Reproduction and Fertility 74, 9-21.

Schultz, R.M., Montgomery, R.R.\& Belanoff, J.R. (1983a) Regulation of mouse oocyte meiotic maturation: implication of a decrease in oocyte cAMP and protein dephosphorylation in commitment to resume meiosis. Developmental Biology 97, 264-273.

Schultz, R.M., Montgomery, R.R., Ward-Bailly, P.F. \& Eppig, J.J. (1983b) Regulation of oocyte maturation in the mouse: possible roles of intercellular communication, cAMP and testosterone. Developmental Biology 95, 294-304.

Seamon, K.B. \& Daly, J.W. (1981) Forskolin: a unique diterpene activator of cyclic AMP-generating systems. Journal of Cyclic Nucleotide Research 7, 201-224.

Seamon, K.B., Padgett, W. \& Daly, J.W. (1981) Forskolin: unique diterpene activator of adenylate cyclase in membranes and in intact cells. Proceedings of the National Academy of Sciences USA 78, 3363-3367.

Tsafriri, A., Linder, H.R., Zor, V. \& Lamprecht, S.A. (1972) In vitro induction of meitoic division in follicle-enclosed rat oocytes by LH, cyclic AMP and prostaglandin $\mathrm{E}_{2}$. Journal of Reproduction and Fertility 31, 39-50.

Yoshimura, Y., Kitai, H., Santulli, R., Wright, K. \& Wallach, E.E. (1985) Direct ovarian effect of clomiphene citrate in the rabbit. Fertility and Sterility 43, $471-476$.

Yoshimura, Y., Hosoi, Y., Atlas, S.J., Bongiovanni, A.M. \& Wallach, E.E. (1986) The effects of ovarian steroidogenesis on ovulation and fertilizability in the in vitro perfused rabbit ovary. Biology of Reproduction 35, 943--948.

Yoshimura, Y., Hosoi, Y., Bongiovanni, A.M., Santulli, R., Atlas, S.J. \& Wallach, E.E. (1987) Are ovarian steroids required for ovum maturation and fertilization? Endocrinology 120, 2555-2561.

Yoshimura, Y., Nakamura, Y., Oda, T., Yamada, H., Nanno, T., Ando, M., Ubukata, Y. \& Suzuki, M. (1990) Effects of gonadotropin-releasing hormone agonists on meiotic maturation of follicle-enclosed oocytes in rabbits. Biology of Reproduction 43, $1012-1018$.

Received 24 June 1991 